\title{
Mesenteric Venous Thrombosis After Laparoscopic Sleeve Gastrectomy: Pathophysiology and Literature Review
}

\author{
Radwan Kassir ${ }^{\mathrm{a}, \mathrm{c}}$, Patrice Lointier ${ }^{\mathrm{b}}$, Christophe Breton ${ }^{\mathrm{a}}$, Pierre Blanc ${ }^{\mathrm{a}}$
}

\begin{abstract}
In the last 10 years, laparoscopic sleeve gastrectomy has become an increasingly popular surgical option for morbidly obese patients. Mesenteric venous thrombosis, as a complication of laparoscopic sleeve gastrectomy, has been rarely reported. We report two cases of thrombosis of the superior mesenteric vein after sleeve gastrectomy. It is confirmed by CT scan. Treatment is primarily medical. Three months later, the patients were asymptomatic still under antivitamin K. Thrombosis of the superior mesenteric vein after bariatric surgery is a diagnosis that one should know how to raise in front of any postoperative abdominal pain. The laparoscopic abdominal pressure $(>14 \mathrm{~mm} \mathrm{Hg})$, associated with a marked position in bariatric surgery, reduces the venous flow by $50 \%$ which may increase the risk of thrombosis. In addition, soft tissue trauma during surgery (manipulation of the small intestine during short gastric bypass or duodenal switch) releases tissue factors that could cause a mesenteric venous thrombosis in patients with a previously undiagnosed hypercoagulable state. The clinical signs of mesenteric venous ischemia are variable and nonspecific. The etiology of portal thrombosis must be done before the introduction of heparin therapy which is not always possible in emergency. An obese patient with a history of thrombosis should receive a complete etiology of these thromboses before bariatric surgery. Abnormal blood dirt, an active smoking fat woman having oral contraceptive, or a patient with a history of recurrent venous thrombosis may be a relative contraindication against a complex bariatric surgery with digestive bypass.
\end{abstract}

Keywords: Thrombosis; Superior mesenteric venous; Laparoscopic sleeve gastrectomy; Diagnosis; Treatment; Pathophysiology

Manuscript accepted for publication May 21, 2014

${ }^{a}$ Department of Digestive Surgery, Clinique Chirurgicale Mutualiste, Saint Etienne, France

${ }^{b}$ Department of Digestive Surgery, Clinique de la Chataigneraie,

Clermont-Ferrand, France

${ }^{\mathrm{c} C}$ Corresponding Author: Radwan Kassir, Department of General Surgery, CHU Hospital, Jean Monnet University, Avenue Albert Raimond, 42270

Saint Etienne, France. Email: Radwankassir42@hotmail.Fr

doi: http://dx.doi.org/10.14740/jcs229w

\section{Introduction}

The first ischemia due to thrombosis in the superior mesenteric vein was reported by Warren and Eberhard in 1935 [1]. This type of venous ischemia represents $5-15 \%$ of all mesenteric ischemia $[1,2]$. We report two cases of thrombosis of the superior mesenteric vein after sleeve gastrectomy, discussing the pathophysiology, the diagnosis, the etiology and treatment from a literature review.

\section{Case Reports}

\section{Case 1}

A 40-year-old patient underwent a laparoscopic sleeve gastrectomy for the treatment of obesity (BMI $41 \mathrm{~kg} / \mathrm{m}^{2}$, decision validated upon multidisciplinary meeting). The patient was an active smoker, had a paired sleep apnea syndrome and a treated depressive disorder. The surgical procedure was simple with an operative time of $60 \mathrm{~min}$. The immediate postoperative course was simple and uneventful, and the hospitalization ended on the fifth day. One month later, the

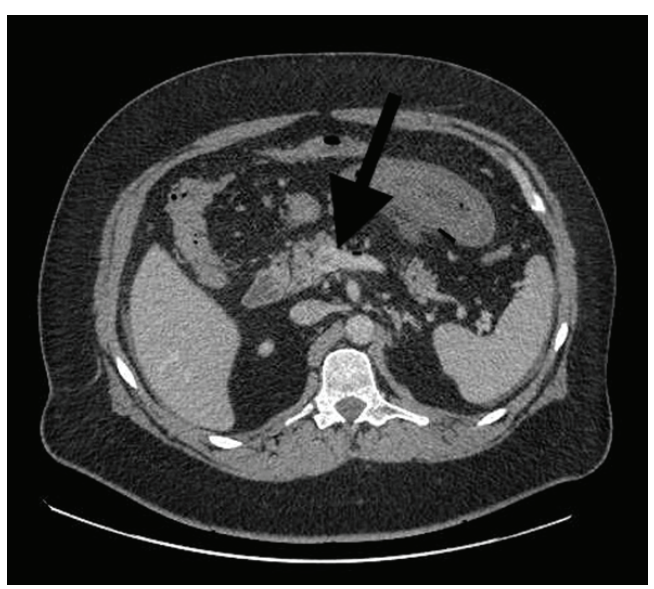

Figure 1. Abdominal CT scan with injected on admission (case 1). Arrow: a thrombosis of the superior mesenteric vein. 


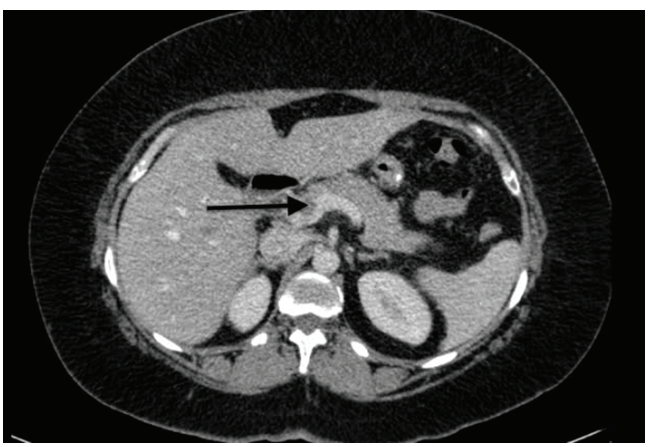

Figure 2. Abdominal CT scan with injected on admission (case 2). Arrow: a portal thrombus at the junction of the superior mesenteric vein and spleno-mesenteric trunk.

patient was asymptomatic and lost $15 \mathrm{~kg}$. The patient was again admitted to emergency on the 40th postoperative day for mild abdominal pain associated with nausea. The abdomen was soft; the patient was afebrile and not tachycardic. The abdomen without preparation found some dilated bowel loops. Biology found a moderate leukocytosis without other abnormalities. The computed tomography (CT) found a thrombosis of the superior mesenteric vein associated to a thickened jejunal loop (Fig. 1). The patient was not left empty stomach. The treatment consisted of intravenous heparin therapy that had a favorable result and a week of hospitalization with the administration of antivitamin $\mathrm{K}$. The etiologiy, made after the introduction of heparin therapy, was negative. The patient was advised to stop smoking. Three months later, the patient was asymptomatic still under antivitamin K. A CT scan control is provided on the sixth postoperative month.

\section{Case 2}

A 47-year-old woman underwent a laparoscopic sleeve gastrectomy for the treatment of obesity (BMI $43 \mathrm{~kg} / \mathrm{m}^{2}$ without comorbidity, decision validated upon multidisciplinary meeting). This patient had a treatment of an oral contraceptive. The laparoscopic sleeve gastrectomy was performed without difficulty, with an operating time of $60 \mathrm{~min}$. The immediate postoperative course was uneventful and the hospitalization ended on the seventh postoperative day. The patient was hospitalized again in emergency, on the seventeenth postoperative day for epigastric pain and left hypochondrium that suddenly appeared 5 days before. It was a transfixing pain, associated with a low-grade fever $\left(38^{\circ} \mathrm{C}\right)$ and chills. The patient was neither tachycardic nor dysphagic nor dyspneic. The abdomen was painful with epigastric defense. Biology found hyperleukocytosis and an increased CRP. A CT scan with injected contrast product found inflammation of the cephalic peripancreatic fat, a portal thrombus at the junction of the superior mesenteric vein and spleno-mesenteric trunk, a permeable portal trunk and no sign of digestive distress (Fig. 2). Oral contraception was stopped; the thrombosis review



Figure 3. An abdominopelvic CT scan control 1 month later (case 2). Arrow: thrombosis on the right portal branch.

was performed prior to the initiation of an anticoagulant treatment that combined a subcutaneously curative low molecular weight heparin and an antivitamin $\mathrm{K}$ treatment. The clinical result was favorable, with pain relief in $48 \mathrm{~h}$. Feeding was resumed after $48 \mathrm{~h}$. During the first postoperative month, the patient did not show any warning digestive sign, with a weight loss of $10 \mathrm{~kg}$. The etiology was negative. An abdominopelvic CT scan control 1 month later showed the disappearance of the portal thrombus in the mesenteric vein, a free and permeable pedicle portal vein, but found thrombosis on the right portal branch (left portal branch permeable), with a right hyperdense liver lobe (Fig. 3). Treatment with vitamin $\mathrm{K}$ was maintained.

\section{Discussion}

The most frequent complications following sleeve gastrectomy are fistula and hemorrhage. Thrombosis of the superior mesenteric vein is exceptional, potentially severe [3]. This type of ischemia has been described after bariatric surgery: sleeve gastrectomy, gastric bypass Y, duodenal switch $[4,5]$. In these cases described above, the diagnosis was made between the 13th and 30th postoperative day. The pathophysiology of thrombosis of the superior mesenteric vein is multifactorial. The laparoscopic abdominal pressure $(>14 \mathrm{~mm}$ $\mathrm{Hg}$ ), associated with a marked position in bariatric surgery, reduces the venous flow by $50 \%$ which may increase the risk of thrombosis. Indeed, hypercapnia induced by $\mathrm{CO}_{2}$ insufflation causes sympathetic vasoconstriction, which increases peripheral resistance, average arterial pressure, pulmonary arterial pressure and pulmonary capillary wedge pressure [6]. In addition, soft tissue trauma during surgery (manipulation of the small intestine during short gastric bypass or duodenal switch) releases tissue factors that could cause a 
mesenteric venous thrombosis in patients with a previously undiagnosed hypercoagulable state [5]. Portal venous flow is affected by increased intraabdominal pressure with carbon dioxide pneumoperitoneum. Finally, obesity itself causes a pro-inflammatory status and an increase in intra-abdominal pressure which are risk factors for venous thrombosis. The steep reverse-Trendelenburg position during laparoscopic bariatric surgery may augment the flow effects of pneumoperitoneum.

The clinical signs of mesenteric venous ischemia are variable and nonspecific. In the presence of abdominal pain of unknown etiology, we shall know how to suggest the diagnosis of a portal vein thrombosis. In case of intestinal ischemia, pain, which is always present, contrasts the absence of physical signs $[7,8]$. It can be associated with nausea, vomiting, diarrhea, high or low gastrointestinal hemorrhage [9]. The presumptive diagnosis is often that of perforated ulcer, or acute pancreatitis [10]. Biology shows leukocytosis in half of patients and elevated lactate in one-third of patients [9]. The hyperleukocytosis was present in the two cases. In obese patients the reference radiological examination is the CT with vascular injection $[11,12]$. In cases of venous thrombosis, it highlights an endovenous hypodense image that does not show contrast (luminal defect related to the presence of the clot) and a thickening of the walls of the small intestine (Fig. 1) [13].

Familiarity with this dangerous entity is important. Prompt diagnosis and care, initiated by a high index of suspicion, is crucial .The treatment of acute intestinal ischemia of venous origin has evolved in the last years [14]. It is now mainly medical. In case of early diagnosis and an abdomen that is "not acute" and presented no infarction, two non-operative treatments may be considered: thrombolysis and systemic heparin $[7,13]$. This anticoagulation treatment should be started only next to hemostasis tests, the thing that was not done in our first case. The activated partial thromboplastin time should be two times the normal [15]. Thrombolytic therapy in the mesenteric system has limited indications [16]. Surgical exploration by laparoscopy is useful in acute abdomens but remains of rare use and is still being discussed in this indication because the hypertension associated with insufflation could theoretically worsen venous ischemia [17]. Surgical exploration consents the lesions assessment with two possible outcomes: 1) in cases of localized intestinal necrosis, treatment consists of a resection and immediate restoration of digestive continuity; 2) when the ischemic or infarcted intestine segment is extended, the limits of resection are difficult to predict. In all cases the resection should be efficient to avoid "short bowel syndrome". Some teams are partisans of a resection followed by a gastrointestinal bypass, associated with an immediate heparin treatment ensued by a second laparotomy 12 - $24 \mathrm{~h}$ later [9]. Finally in case of venous infarction, it is possible to perform a surgical method halfway between the Thiry-Vella technique and Mulholland technique (high jejunostomy). The idea is to put to rest the ischemic intestine, performing either a double exclusionary stoma brought up (jejunostomy) and taken down (ileostomy) is a simple high stoma. These techniques are then used to monitor the intestinal vitality by the appearance of the stoma. In cases already recently described after bariatric surgery, treatment was medical in five cases and chirurgical in one case (intestinal resection in the aftermath of a $\mathrm{Y}$ gastric bypass).

The etiology of portal thrombosis must be done before the introduction of heparin therapy which is not always possible in emergency (case 1). This assessment is important because $20-35 \%$ of thrombosis results from primary causes [18]. The results should include local factors (intra-abdominal infection or malignancy), general factors (constitutional deficits in protein $\mathrm{C}$, protein $\mathrm{S}$ and antithrombin III, a myeloproliferative disorder that can be latent) [19]. It was negative in both our cases, but in the first case, etiology was made after the introduction of heparin therapy that could skew the results. Prognostic factors of these venous thromboses are early diagnosis, etiology, scope and speed of thrombosis installation and early treatment $[16,19]$. In case of active smoking and being on an oral anti-contraception treatment, their cessation must be affirmed to the patient when done.

Thrombosis of the superior mesenteric vein after bariatric surgery is a diagnosis that one should know how to raise in front of any postoperative abdominal pain. It is confirmed by CT scan. Treatment is primarily medical. An obese patient with a history of thrombosis should receive a complete etiology of these thromboses before bariatric surgery. Abnormal blood dirt, an active smoking fat woman having oral contraceptive, or a patient with a history of recurrent venous thrombosis may be a relative contraindication against a complex bariatric surgery with digestive bypass.

\section{Conflict of Interests}

No conflict of interests.

\section{References}

1. Warren S, Eberhard TP. Mesenteric venous thrombosis. Surg Gynecol Obstet. 1935;61:102-121.

2. Ottinger LW, Austen WG. A study of 136 patients with mesenteric infarction. Surg Gynecol Obstet. 1967;124(2):251-261.

3. Swartz DE, Felix EL. Acute mesenteric venous thrombosis following laparoscopic Roux-en-Y gastric bypass. JSLS. 2004;8(2):165-169.

4. Gandhi K, Singh P, Sharma M, Desai H, Nelson J, Kaul A. Mesenteric vein thrombosis after laproscopic gastric sleeve procedure. J Thromb Thrombolysis. 
2010;30(2):179-183.

5. Johnson CM, de la Torre RA, Scott JS, Johansen T. Mesenteric venous thrombosis after laparoscopic Roux-enY gastric bypass. Surg Obes Relat Dis. 2005;1(6):580582; discussion 582-583.

6. Haglund U, Norlen K, Rasmussen I, et al. (1995). Complications related to pneumoperitoneum. In: Bailey RW, Flowers JL, eds. Complications of Laparoscopic Surgery. St. Louis: Quality Medical Publishing Inc: 45-8.

7. Cohen J, Edelman RR, Chopra S. Portal vein thrombosis: a review. Am J Med. 1992;92(2):173-182.

8. Abdu RA, Zakhour BJ, Dallis DJ. Mesenteric venous thrombosis--1911 to 1984. Surgery. 1987;101(4):383388.

9. Rhee RY, Gloviczki P, Mendonca CT, Petterson TM, Serry RD, Sarr MG, Johnson CM, et al. Mesenteric venous thrombosis: still a lethal disease in the 1990s. J Vasc Surg. 1994;20(5):688-697.

10. Case records of the Massachusetts General Hospital. Weekly clinicopathological exercises. Case 51-1984. A 45-year-old man with abdominal pain and abnormal CT scan. N Engl J Med. 1984;311(25):1622-1628.

11. Parvey HR, Raval B, Sandler CM. Portal vein thrombosis: imaging findings. AJR Am J Roentgenol. 1994;162(1):77-81.

12. Verbanck JJ, Rutgeerts LJ, Haerens MH, Tytgat JH, Segaert MF, Tytgat HJ, Afschrift MB. Partial splenoportal and superior mesenteric venous thrombosis. Early sonographic diagnosis and successful conservative management. Gastroenterology. 1984;86(5 Pt 1):949-952.

13. Bradbury AW, Brittenden J, McBride K, Ruckley CV. Mesenteric ischaemia: a multidisciplinary approach. $\mathrm{Br}$ J Surg. 1995;82(11):1446-1459.

14. Delpero JR (1995). Ischemies aigues et infarctus enteromesenteriques. Dans : Ducerf C, Laurian C. Pathologie vasculaire du tube digestif. Monographie de l'AFC. Paris: Arnette Blackwell, 145-72.

15. Joh JH, Kim DI. Mesenteric and portal vein thrombosis: treated with early initiation of anticoagulation. Eur J Vasc Endovasc Surg. 2005;29(2):204-208.

16. Poplausky MR, Kaufman JA, Geller SC, Waltman AC. Mesenteric venous thrombosis treated with urokinase via the superior mesenteric artery. Gastroenterology. 1996;110(5):1633-1635.

17. Henry C, Smadja C, Vons C, Bobocescu E, Mariette D, Tahrat M, Franco D. [Results of laparoscopic treatment of abdominal emergencies]. Ann Chir. 1998;52(3):223228.

18. Bayraktar Y, Harmanci O. Etiology and consequences of thrombosis in abdominal vessels. World J Gastroenterol. 2006;12(8):1165-1174.

19. Lefrancois C, Derlon A, Le Querrec A, Justum AM, Gautier P, Maurel J, Leroux Y, et al. [Mesentric venous thrombosis. Risk factors, treatment and outcome. An analysis of 18 cases]. Ann Fr Anesth Reanim. 1994;13(2):182-194. 